\title{
IR Imaging of Spiral Galaxy Bulges
}

\author{
DENNIS ZARITSKY \\ Carnegie Observatories, 813 Santa Barbara St., Pasadena, CA, 91101, U.S.A. \\ MARCIA RIEKE \\ Steward Observatory, Univ. of Arizona, Tucson, AZ, 85721, U.S.A. \\ and \\ HANS-WALTER RIX \\ Institute for Advanced Study, Princeton, NJ, 08540, U.S.A.
}

\begin{abstract}
Imaging in the infrared $(2.2 \mu)$ minimizes the impact of dust obscuration and allows reliable mapping of the mass-tracing stellar population in spiral galaxies. We find dramatic differences compared to photometry at shorter wavelengths (e.g. $0.8 \mu$ ). As an example, the observations of the mini-bar and inner spiral arms of M 51 are discussed.
\end{abstract}

Key words: galaxies: photometry - galaxies: spiral - galaxies: individual: M 51

The apparent shape of the disk and bulge components of spiral galaxies is best studied through infrared imaging because distortions of the light from the masstracing stellar population by light from young stars and by dust are minimized. Imaging face-on galaxies eliminates complications from projection effects. Although our project consists of a systematic study of about 25 galaxies with inclinations of less than $10^{\circ}$, in this paper we only discuss preliminary results from observations of M 51 .

The data presented here were obtained with the Steward Observatory $2.3 \mathrm{~m}$ telescope using either a CCD (B and I images, 0.45 and $0.8 \mu$ respectively) or a Nicmos $3(256 \times 256)$ array $(\mathrm{K}$ images, $2.2 \mu)$. Exposure times range from $2 \mathrm{~min}$ (CCD) to $15 \mathrm{~min}$ (IR) and the data were reduced using standard techniques.

The oval distortion seen at I by Zaritsky and Lo (1986) and Pierce (1986) is found to be much more prominent at $2.2 \mu$. Whether this distortion is a prolate bulge or a bar is an observationally subtle distinction, which may, however, imply different origins. Based on the IR surface photometry, we argue that the oval distortion is a bar, because the spiral arms appear to begin at the edges of this feature (cf. Figure 1 in which a smooth model has been subtracted from the K-band image) and because a power-law fit to the intensity profile in the inner region overpredicts light at large radii (i.e. there must be a distinct truncation of the oval component). However, the distinction between bar and oval bulge is not unambiguous.

Pierce (1986) also claimed to have observed an inner ring of material at the edge of the bulge. We cannot comment on the possible presence of a gaseous ring, but we do not observe a stellar ring. Instead we see the continuous winding of spiral arms to the edge of the bar. Comparison of $\mathbf{J}$ and $\mathrm{K}$ images shows that these arms are primarily ridges of stellar emission, not contrast enhancements from dust lines. Since the arms are tightly wound these could have been mistaken for a ring. This distinction can only be made with the K-band images. Models of the interaction between M 51 and its companion suggest that the spirals arms, at least at large radii, are material arms generated by tidal interaction (Hernquist 1990). However, if the inner arms are material arms as well we can use the winding of the arms to estimate 


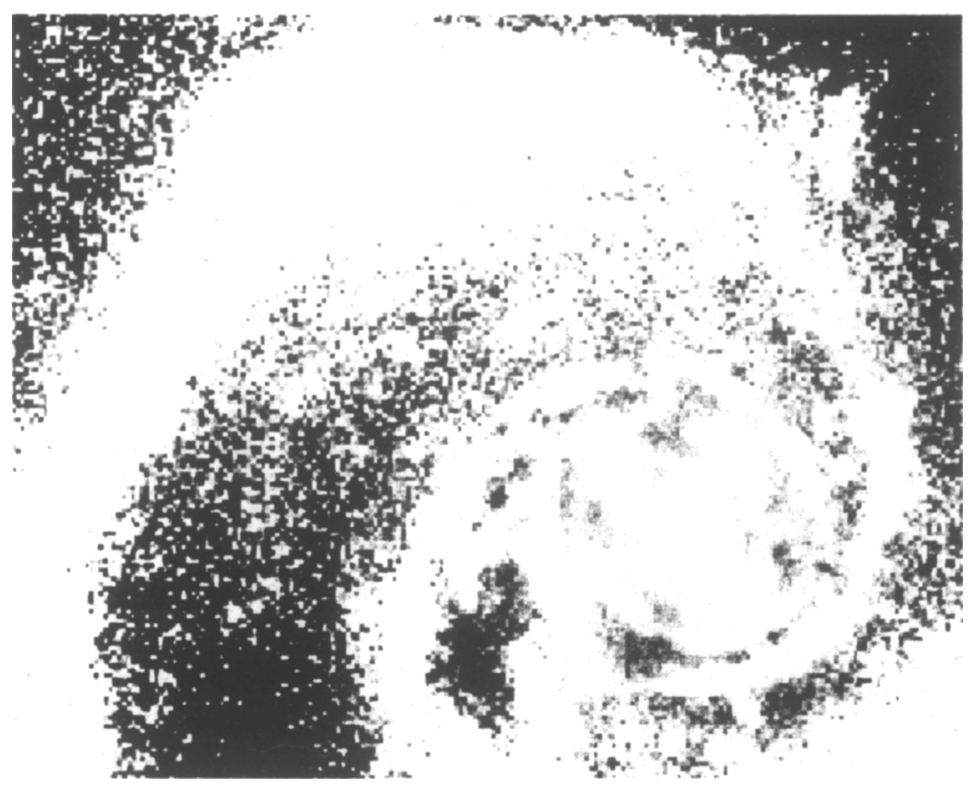

Fig. 1. Model-Subtracted K-Band Image of Inner Region of M 51

the time since their formation (cf. Binney and Tremaine 1987). We find that if the rotation curve is presumed to be flat with a value of $200 \mathrm{~km} \mathrm{sec}^{-1}$ (Tully 1974, Goad et al. 1979) then the implied age $\left(2.3 \times 10^{7} \mathrm{yrs}\right)$ is inconsistent with the estimated time since the pericenter passage of NGC $5195\left(\sim 3 \times 10^{8}\right.$ yrs; Hernquist 1990). Unfortunately, the shape of the rotation curve is not sufficiently well determined to place strong constraints. The continuity of these arms through the inner Lindblad resonance, their thinness, and their tight winding are remarkable.

\section{Acknowledgements}

D.Z. and H.-W.R. acknowledge support through Hubble Fellowships from STScI under contract to NASA (HF-1027.01-91A and HF-1027.01-91A respectively). D.Z. thanks the IAS for its hospitality during his visit. We gratefully acknowledge financial support for the infrared camera from the National Science Foundation and NASA for providing the Nicmos array.

\section{References}

Binney, J. and Tremaine, S. (1987), Galactic Dynamics, (Princeton University Press: Princeton). Goad, J.W., DeVeny, J.B., and Goad, L.E. (1979), Ap. J. Suppl., 39, 439.

Hernquist, L. (1990), in Dynamics and Interactions of Galaxies, ed. R. Wielen, (Heidelberg: Springer-Verlag), p. 108.

Pierce, M.J. (1986), A.J., 92, 285.

Tully, R.B. (1974), Ap. J. Suppl., 27, 449.

Zaritsky, D., and Lo, K.-Y. (1986), Ap. J., 303, 66. 